\title{
A PRELIMINARY STUDY ON CALCULATION METHOD OF VISUAL IMPACT OF URBAN ARCHITECTURAL HERITAGE
}

\author{
Shuo Yang ${ }^{1,2 *}$, Yungang $\mathrm{Hu}^{1.2}$, Miaole $\mathrm{Hou}^{1.2}$ \\ ${ }^{1}$ School of Geomatics and Urban Spatial Informatics, Beijing University of Civil Engineering and Architecture, No.15 Yongyuan \\ Road, Daxing District, Beijing; 102616, China; bucea ys@126.com (S.Y); huyungang@bucea.edu.cn (Y.H.); \\ houmiaole@bucea.edu.cn (M.H.). \\ ${ }^{2}$ Beijing Key Laboratory for Architectural Heritage Fine Reconstruction and Health Monitoring, No.15 Yongyuan Road, Daxing \\ District, Beijing; 102616, China
}

KEY WORDS: Urban architectural heritage, Skyline analysis, Visual impact, Quantitative calculation, The Lama Temple

\begin{abstract}
:
Urban architectural heritage as a part of cultural heritage, its surrounding buildings will have a certain visual impact on the cultural heritage itself. This paper presents a method for calculating visual impact and applies it to the field of urban architectural heritage. In this method, the visual radius and visual range are calculated based on the theoretical knowledge related to the range of human eye line of sight and the curvature of the earth, and then the visual projection plane is established. Then, through skyline analysis and spatial relationship calculation, the urban architectural heritage and its surrounding buildings are projected. Calculate the visual impairment index through the projection area and the projection surface area to quantify the visual impact. By calculating the visual impact in The Lama Temple area, it is proved that the method presented in this paper is effective and advanced in practical application.
\end{abstract}

\section{INTRODUCTION}

In 2015, the United Nations Sustainable Development Summit held in New York adopted 17 Sustainable Development Goals (SDGs), and its sub-goal 11.4 "Further efforts to protect and defend the world's cultural and natural heritage" mentioned Sustainable development of cultural heritage (Chen et al, 2019). As an important category of cultural heritage, architectural heritage has extremely high historical and cultural value, so it must be protected and inherited. For this reason, it is particularly important to evaluate the sustainable development status of architectural heritage. Aiming at the evaluation of the Sustainable Development Goals, Chen Jun and others used geographic information technology and combined statistical data and geographic information data to preliminarily study the quantitative evaluation methods of the Sustainable Development Goals (Chen et al, 2019; Chen et al, 2018).

As a method of evaluating the degree of impact of outstanding universal value (OUV) of cultural heritage, heritage impact evaluation has many similarities with the evaluation of the sustainable development status of cultural heritage. It is mentioned in the heritage impact evaluation that the authenticity and completeness of cultural heritage are important aspects of outstanding universal value. Regarding the integrity of cultural heritage, Sun Yan proceeded from visual integrity to evaluate the impact of the surrounding environment of many world heritage sites on the world heritage site itself (Sun, 2018). Baharak Seyedashrafi and others also analysed the visual impact of the surrounding buildings of the Cologne Cathedral on itself (Baharak, 2019). It can be seen that the evaluation of visual impact is very important in evaluating the sustainable development status of cultural heritage. In this article, visual impact refers to the environment surrounding the cultural heritage, mainly buildings, which damage the visual integrity of the cultural heritage, as shown in Figure 1 and Figure 2.

In recent years, most domestic scholars have started from the discipline of urban planning to quantitatively evaluate the openness of built-up space (Niu and $\mathrm{Xu}, 2011$ ), and at the same time quantitatively analyse the urban skyline based on visual impact (Niu and Li, 2013), but the related research is mostly based on linear. The analysis of ground features has not been conducted based on the analysis of surface features or a certain area. Most foreign scholars use wind power plants as the research object to analyse their visual effects (Theocharis et al, 2009), and there are few studies related to cultural heritage.

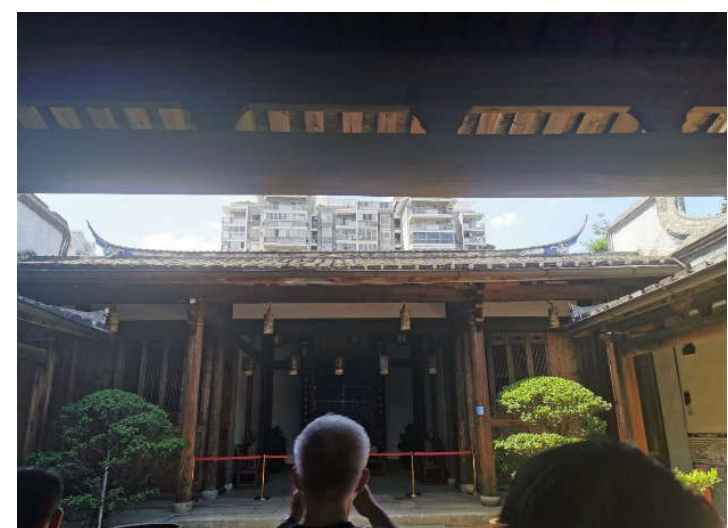

Figure 1 An architectural heritage and its surrounding environment in a city in southern China

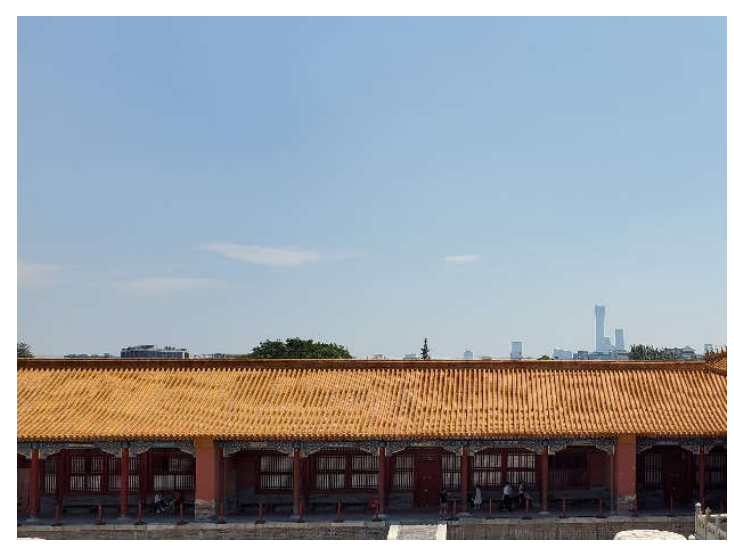

Figure 2 An architectural heritage and its surrounding environment in a city in northern China

\footnotetext{
* Corresponding author: E-mail address: bucea_ys@126.com
} 
Based on the relevant theories of sustainable development, this paper combines the outstanding universal value of cultural heritage and heritage impact evaluation, and proposes the indicator of visual impact. By evaluating the visual impact of urban architectural heritage, it provides an indicator for evaluating the sustainable development status of urban architectural heritage.

\section{VISUAL RANGE CALCULATION}

\subsection{Visual radius calculation}

As we all know, the human eye line of sight is a straight line extending horizontally outwards, while the plane swept by the line of sight during a week's rotation is a horizontal plane. In geodesy theory, the use of horizontal surface instead of level is a limit, in the exceeding of certain limits, the elevation, distance, etc. have an impact (Kong et al, 2010), this paper only considers the impact on elevation.

Because the earth is an irregular spheroid, and the existence of the earth curvature, and the elevation measurement has a certain impact, called the elevation error caused by the curvature of the earth, usually expressed in $\Delta h$, as shown in Figure 3.

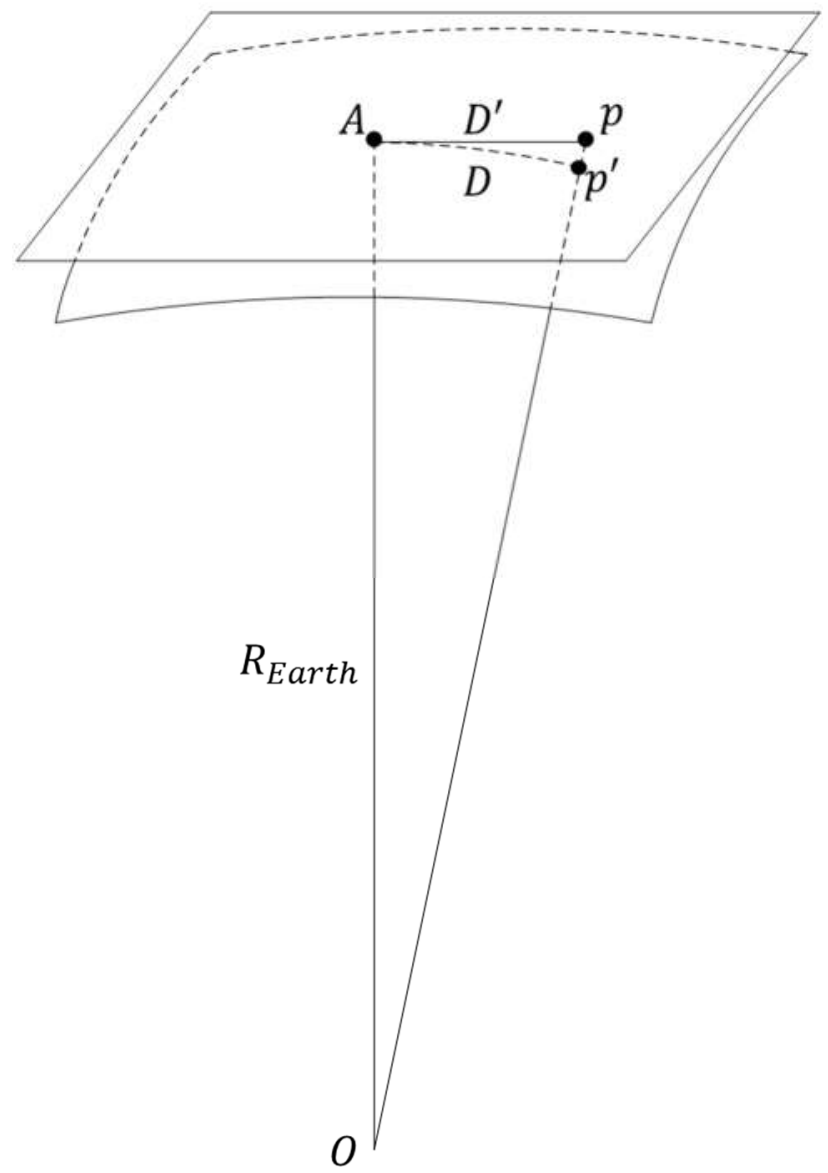

Figure 3 Schematic diagram of the elevation error caused by the curvature of the earth (pp')

the specific calculation is as follows:

$$
\begin{gathered}
\left(R_{\text {Eart } h}+\Delta h\right)^{2}=R_{\text {Eart } h}^{2}+D^{2} \\
\Delta h=\frac{D^{2}}{2 R_{\text {Earth }}+\Delta h}
\end{gathered}
$$

Since $\Delta h$ is small relative to $2 R$, the $\Delta h$ in the denominator can be ignored until the final formula is calculated:

$$
\Delta h=\frac{D^{2}}{2 R_{\text {Eart } h}}
$$

where

$$
R_{\text {Earth }}=\text { earth radius }
$$

$D=$ the horizontal distance between two points on the

ground

For different distances, the resulting curvature error for the earth is shown below:

\begin{tabular}{|c|l|l|l|l|l|}
\hline$D(\mathrm{~m})$ & 10 & 50 & 100 & 150 & 200 \\
\hline$\Delta h(\mathrm{~mm})$ & 0.00 & 0.20 & 0.80 & 1.77 & 3.10 \\
\hline
\end{tabular}

Table 1. Elevation error caused by the curvature of the earth at different distances.

The above calculations show that the curvature of the earth has a greater effect on elevation, even at a distance of not too long such as $150 \mathrm{~m}$, the elevation has an error of $1.77 \mathrm{~mm}$. Therefore, in the study related to elevation, it is not easy to use the horizontal surface instead of the horizontal surface, to consider the impact of the curvature of the earth.

In calculating the visual radius, the eye is viewed from a distance when the eye is standing upright, forming a horizontal line of sight, and investigates the average height of the buildings in the area where the urban architectural heritage is located, and calculates the visual radius based on the distance between the horizontal line of sight and the building at this height.

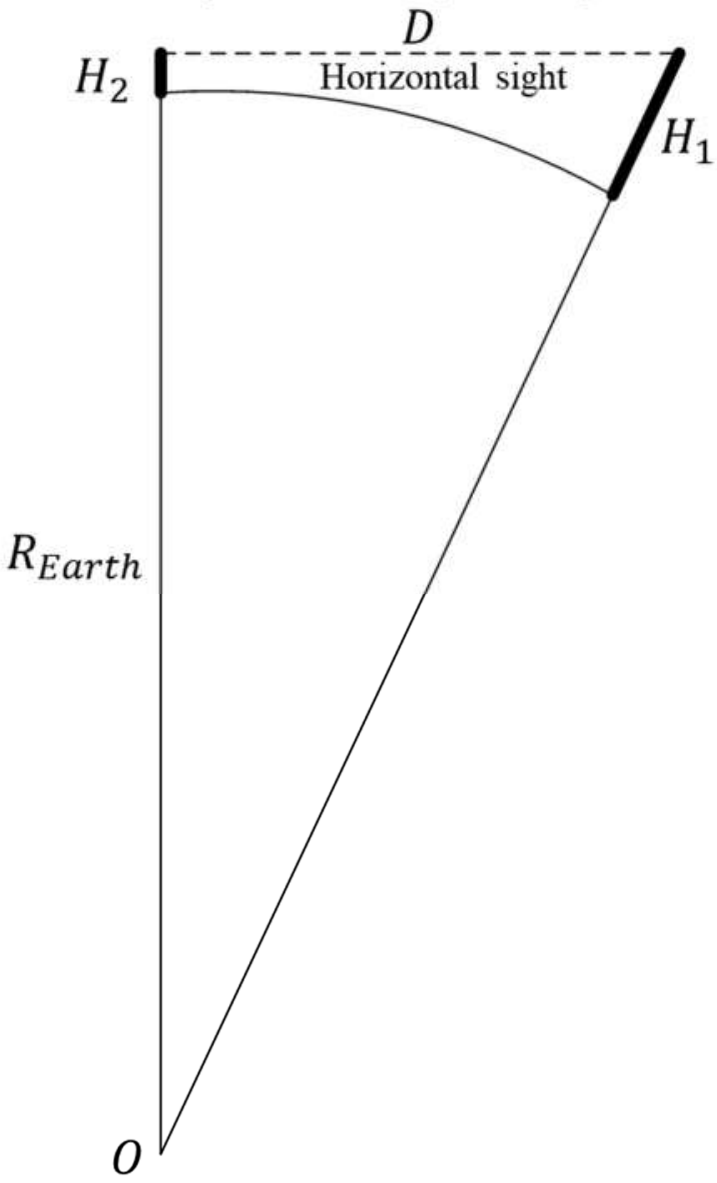

Figure 4 Schematic diagram of visual radius calculation

As can be seen from the figure:

So:

$$
\left(H_{1}+R_{\text {Earth }}\right)^{2}=\left(H_{2}+R_{\text {Earth }}\right)^{2}+D^{2}
$$

$$
D=\sqrt{\left(H_{1}-H_{2}\right)\left(2 R_{\text {Earth }}+H_{1}+H_{2}\right)}
$$

where $H_{1}=$ average height of the buildings 
ground

$\mathrm{H}_{2}=$ the linear distance between two points on the

$$
R_{E a r t h}=\text { earth radius }
$$

\subsection{Visual space construction}

When a person looks at the outside world, his field of view is usually a hemisphere (Yang et al, 2007). Thus, according to the calculated visual radius, the hemispheric body is established for the centre of the urban architectural heritage area, that is, the field of view space, and the hemispherical surface is the projection surface (Figure 5).

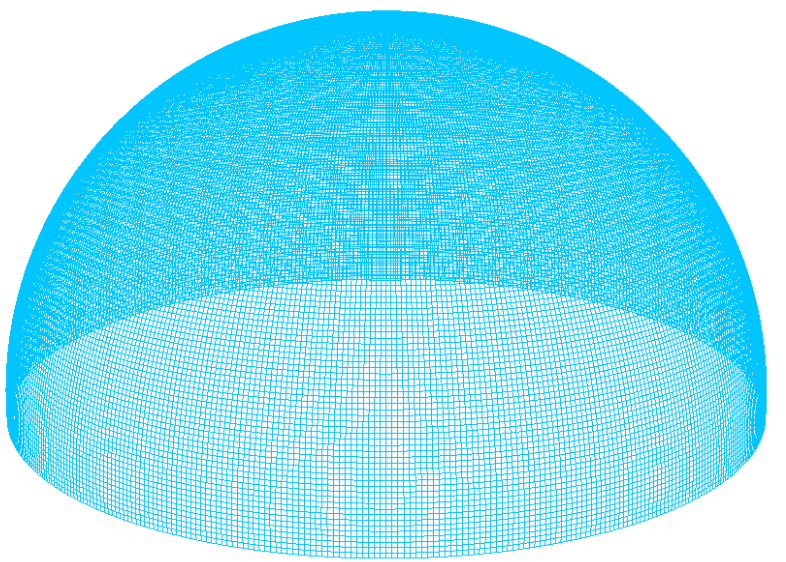

Figure 5 Schematic diagram of view space and projection surface

\section{VISUAL IMPAIRMENT CALCULATION}

\subsection{Visual impairment projection}

The visual impairment mentioned in this article refers to the visual impairment of the area of the building after the projection of the building in this direction when the two boundary points at the highest point of view of the surrounding building intersect. Skyline analysis, as a common 3D spatial analysis tool, is commonly used to analyse spatial visibility (Yu et al, 2008). The skyline, also known as the outline or panoramic view of the city, is the overall structure of the tall buildings in the city, or the local landscape consisting of many skyscrapers. In ArcGIS, the skyline tool generates a 3D line that is the boundary between the sky and the surfaces around the observation points and features.

After the skyline is calculated, each vertex of the skyline is obtained by spatial relationship calculation and projected onto the projection surface described above, as follows (Figure 6):

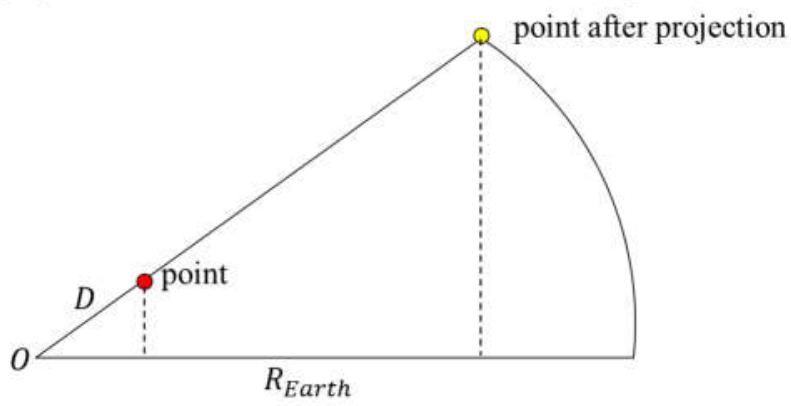

Figure 6 Satellite images of The Lama Temple and its surrounding environment (partial)

(1) Establish a spatial right-angle coordinate system with the viewpoint as the origin $(0,0,0)$;
(2) Calculate the Euclidean Distance $D$ from each vertices $\left(x_{i}, y_{i}, z_{i}\right)$ to the origin, the formula is as follows:

$$
D=\sqrt{\left(x_{i}-0\right)^{2}+\left(y_{i}-0\right)^{2}+\left(z_{i}-0\right)^{2}}
$$

(3) Calculate the ratio of the distance from each vertex to the origin to the radius $R$ (visual radius) of the hemisphere, and obtain the scale factor $k$, the formula is as follows:

$$
k=\frac{R}{D}
$$

(4) Scale by calculated scale factor to get the coordinates of the points after projection $\left(x_{i}^{\prime}, y_{i}^{\prime}, z_{i}^{\prime}\right)$

Connect two adjacent points after projection, and each point is a vertical line downward along the projection surface, and the enclosed area is the visual occlusion area.

\subsection{Visual impairment projection area calculation}

Since the visual impairment area is composed of a spherical quadrilateral, it is not an ordinary planar tetrahedron, so it is necessary to use the formula for calculating spherical polygons in spherical geometry to calculate the area of each visual occlusion area, the formula is as follows (Liu, 2005):

$$
S=R^{2}\left[\sum_{i=1}^{n} \alpha_{i}-(n-2) \pi\right]
$$

where $\quad R=$ the radius of the sphere

$n=$ the number of sides of a spherical polygon

$\alpha_{i}=$ the $i$-th interior angle of a spherical polygon

Sum the calculated area of each visual occlusion area to get the final visual occlusion area, the formula is as follows:

$$
S_{\text {visual impairment }}=\sum_{i=1}^{m} S_{i}
$$

where $\quad m=$ the number of visual impairment areas

The method proposed in this paper holds that when performing visual occlusion calculation, only visual masking in urban architectural heritage area is considered first, which is called the lower limit of visual impairment. Then, remove the urban architectural heritage area and consider only the visual occlusion of the building in the viewport space, called the visual impairment upper limit. Overlay the two to get the final visual masking area.

This paper proposes the visual impairment index $c_{\text {visual impairment }}$ to calculate the visual impact, that is, to calculate the proportion of the visual occlusion area on the projection surface, the formula is as follows:

$$
\begin{aligned}
c_{\text {visual impairment }} & =\frac{S_{\text {visual impairment }}}{S_{\text {projection surface }}} \times 100 \% \\
\text { where } \quad \mathrm{S}_{\text {visual impairment }} & =\text { the area of the visual impairment } \\
\mathrm{S}_{\text {projection surface }} & =\text { the area of the projection surface }
\end{aligned}
$$

\section{CASE STUDY AND DISCUSSION}

Taking The Lama Temple, the first batch of The National Key Cultural Relics units as an example, this paper makes a case study of the method proposed in this paper, and then verifies the feasibility and accuracy of the method.

Using the vectorized results of The Lama Temple and its surrounding buildings and combining the height data (Figure 7), The Lama Temple and its surrounding buildings are stretched into a three-dimensional model (Figure 8) to provide a data basis for subsequent experiments. 


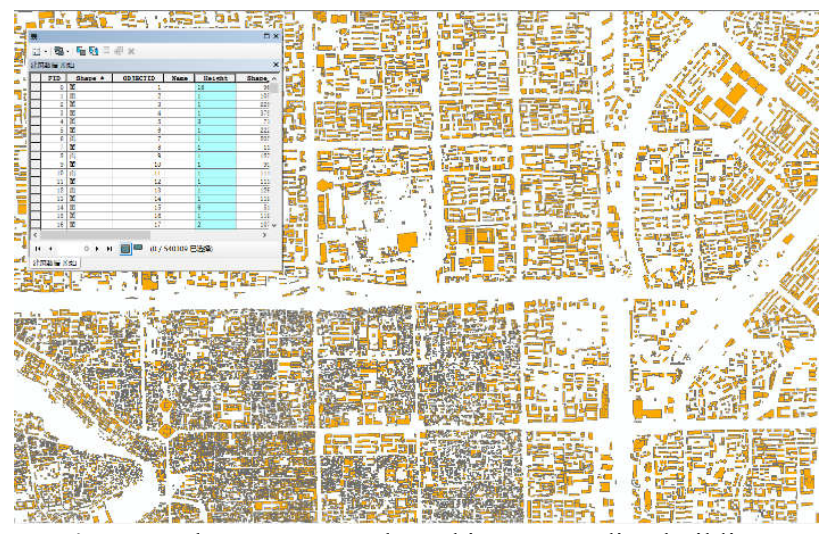

Figure 7 The Lama Temple and its surrounding buildings vector diagram (partial)

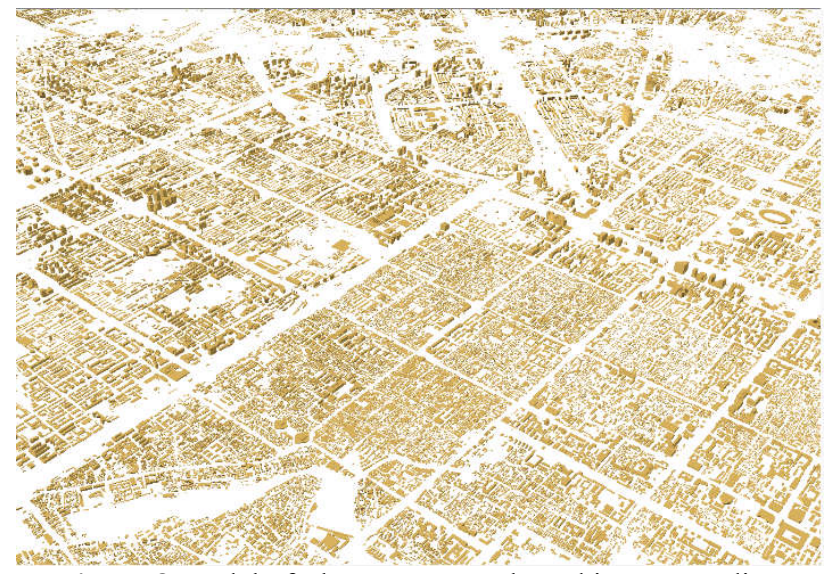

Figure 8 Model of The Lama Temple and its surrounding buildings (partial)

By looking at the relevant information, it can be seen that the average height of buildings in the Beijing area where The Lama Temple is located is about $50 \mathrm{~m}$ (Wang and $\mathrm{Xu}, 2018$ ). According to 'Report on Nutrition and Chronic Disease Status of Chinese Residents (2020)' (Liu, 2020), the average height of men and women aged $18-44$ in China is $1.69 \mathrm{~m}$ and $1.58 \mathrm{~m}$, respectively. According to formula (2), the theoretical value of the visual radius of The Lama Temple area is calculated to be about 24859 $\mathrm{m}$. Combined with the research and the actual situation, it was finally determined that the visual radius of The Lama Temple area was $10000 \mathrm{~m}$.

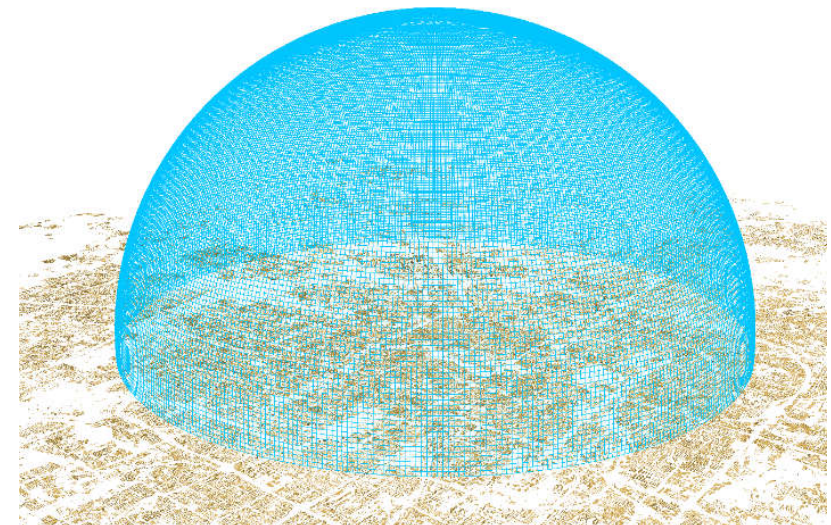

Figure 9 The view space and projection surface of The Lama Temple

Using ArcGIS and AutoCAD to perform skyline analysis and spatial relationship calculation, calculate the lower limit of visual impairment and the upper limit of visual impairment respectively and calculate the visual impairment index.

Through the above experiments, it can be seen that as The Lama Temple located in the Beijing, its visual impairment control is better. According to the results, it can be considered that the height control of the buildings around The Lama Temple is better, and the visual masking problem is lighter. For those who visit the Summer Palace, there will be a better experience.

\section{CONCLUSION}

By the method proposed in this paper, the visual radius is calculated, the field of view space is established, and the visual impairment index is calculated, which can realize the visual impairment calculation for the urban architectural heritage, and quantify the visual impact of the urban architectural heritage to a certain extent. At the same time, visual impairment index, as an index to evaluate visual impact, also lays a certain foundation for the subsequent visual impact evaluation of urban architectural heritage.

In subsequent studies, we can consider a comprehensive multiview analysis, a more in-depth study of other factors of visual impact, such as the shape and color of buildings around urban architectural heritage, etc., will be a comprehensive analysis of the two, so as to more systematically analyse the visual impact of urban architectural heritage.

\section{ACKNOWLEDGEMENTS (OPTIONAL)}

This research was supported by the National Natural Science Foundation of China (NSFC) (Key Project \#41930650) and Youth Beijing Scholar Program.

\section{REFERENCES}

Chen, J., Peng, S., Zhao, X.S., Ge, Y.J., Li, Z.L., 2019. Measuring regional progress towards SDGs by combining geospatial and statistical information. Acta Geodaetica et Cartographica Sinica. 2019, 48(04), 473-479.

Chen, J., Ren, H.R., Geng, W., Peng, S., Ye, F.H., 2018. Quantitative Measurement and Monitoring Sustainable Development Goals (SDGs) with Geospatial Information. Geomatics World. 2018, 25(01), 1-7.

Sun, Y., 2018. A Brief Analysis of the Integrity Evaluation and Protection of World Cultural Heritage from the Perspective of Visual Integrity. China Cultural Heritage. 2018(01), 9-16.

Baharak, S., Xu, Z.L.(Translate), 2019. Effective Contribution of Heritage Impact Assessment to the Conservation of World Heritage Sites: World Heritage Properties of Cologne Cathedral and the Historic Centre of Vienna. World Architecture. 2019(11), 56-67+138.

Niu, X.Y., Xu, F., 2011. Quantitative evaluation of Spatial Openness of Built Environment Using Visual Impact Analysis. Urban Planning Forum. 2011(01), 91-97.

Niu, X.Y., Li, K.K., 2013. A Quantitative Approach to Visual Impact Analysis of City Skyline. Urban Planning Forum. 2013(03), 99-105.

Theocharis, T., Androniki T., Manolis T., Michalis S., 2009. Visual impact evaluation of a wind park in a Greek island. Applied Energy. 86(4), 546-553. DOI: 10.1016/j.apenergy.2008. 08.013 . 
Kong, X.Y., Guo, J.M., Liu, Z.Q., 2010: Foundation of Geodesy. Wuhan University Perss, China.

Yang, P.P., Putra, S.Y., Li, W.J. 2007. Viewsphere: a GIS-based 3D visibility analysis for urban design evaluation. Environment and Planning B: Planning and Design. 34(6), 971-992. DOI: $10.1068 / \mathrm{b} 32142$.

Yu, K.J., Xi, X.S., Wang, S.S., 2008. Urban Landscape Planning Based on Ecological Infrastructure _ A Case Study of Urban Landscape in Weihai City, Shandong Province. City Planning Review. 2008(03), 87-92.

Liu, S.Z., 2005. Spherical polygon area. Journal of Continuing Higher Education. 2005(01), 25-26+30.

Wang, Y., Xu, B.Y., 2018. The Construction of OrderTaking the 'Planning Plan for Building Height Control in the Central City of Beijing' as an example. Beijing Planning Review. 2018(02), 50-57.

Liu, Y.J., 2020. "Report on Nutrition and Chronic Disease Status of Chinese Residents (2020)" Released. Food and Nutrition in China. 2020, 26(12), 2. 